\title{
PROCESS MANAGEMENT AND CONTROL FOR HETEROGENEOUS DOMAIN MODELS
}

\author{
Takeshi MASUDA \\ NTT Access Network Service Systems Laboratories
}

\begin{abstract}
The workflow of telecommunication operation support systems (telecomOSSs), which are a typical example of a heterogeneous domain model, often involves complicated cancellation processing. This paper proposes a ruledriven cancellation method and a middleware component that implements the method. They separate the cancellation process from the workflow and enable the OSSs to be made more flexible.
\end{abstract}

Key words: process management, domain model, workflow, access network

\section{INTRODUCTION}

The diversification of communication services and the shortening of release periods have necessitated the use of commercial off-the-shelf (COTS) software for telecommunication operation support systems (telecom OSSs). Furthermore, when constructing a system that consists of multiple subsystems and that executes operations across all of the operation menus provided by the subsystems, the application of a workflow management system (WfMS) has been found to be effective [1].

When the subsystems developed for each domain are interconnected and the domain models for state transition and cancellation operations of the subsystems differ, cooperative processing becomes difficult. Because it is not always possible to easily change the domain models, the WfMS must be made highly flexible to enable different models and systems to cooperate through it. However, the scale and complexity of the workflow description increases when the WfMS has to handle exceptions and failures [2].

In this paper, we introduce a telecom-OSS, as a typical example of a system that has several subsystems with different domain models. We describe the problem of processing the telecom-OSS workflow. In Section 1, we show that in telecom-OSSs, this problem becomes even more serious than in a typical business flow. In section 2 , we describe a middleware component we developed that implements a rule-driven

The original version of this chapter was revised: The copyright line was incorrect. This has been corrected. The Erratum to this chapter is available at DOI: 10.1007/978-0-387-35674-7_66 
cancellation method to handle exceptions and failures in workflow processing. We simulated this system and found that the total number of activity nodes on practical workflows can be reduced roughly by $50 \%$.

\section{PROCESSING THE TELECOM-OSS WORKFLOW}

Telecommunication operation support systems need a workflow more complicated than that of a general business process mainly because a telecom-OSS domain consists of various related subdomains. This variety arises from many differences among the subdomains, e.g., in the network structure, equipment type, and service classification. This paper describes a system for access network element allocation (ANEA), which is an example of a telecom-OSS. The ANEA domain consists of three subdomains: (1) an outside cable, (2) an intra-office cable, and (3) access equipment (Figure 1 (a)). The internal state of each subsystem changes from an initial state to a completion state. There are also two interim states: allocated and reserved. Each subsystem has several operation menus to initiate forward processes, which move the subsystem to the next state. The host system executes the menus of the subsystems in a certain order; it also manages and controls the whole process. When the host system changes a subsystem's state back from an interim state to the initial state, a cancellation process must be performed. These transitions are modeled as the optimal transition within the subdomain. To be more precise, each ANEA subdomain needs a different amount of time to provide network elements. For example, the outside-cable subdomain needs several weeks, but the access equipment subdomain needs several seconds. Therefore, the access equipment subdomain has only one interim state (allocated), while the other subdomains have two interim states (allocated and reserved).

Figure 1 shows the effect of adding a subdomain (division or subsystem) to an ANEA system and a typical business processing system on the processing order of these systems' workflow. The processing order of a typical business flow (Figure 1 (b)) is affected only by the stable and organized order in the whole domain, e.g., the order of the official sanctioning. It is clear from the figure that the change of the order is local, and the addition of the subdomain is easy: only one flow should be added. However, adding a new subdomain (subsystem) to the ANEA system affects the ANEA workflow (Figure 1 (a)), which makes the addition all the more difficult: in this case, five flows should be added. This is because the priority between the agreement level and the subdomains is inverted. Therefore, the processing order of the ANEA flow is inherently more complicated than that of the typical business flow.

On the other hand, when viewed from the perspective of all the subsystems, the processing order consists of several phases. The internal states of all the subsystems should be synchronized to guarantee macro data consistency. The important point is that the number of times the state of each subsystem changes may differ according to the subdomain model. In this paper, these subdomain models are referred to as heterogeneous domain models (HDMs). The complexity of the HDM workflow adversely affects the process of operation cancellation. When the user cancels an operation or the system generates an exception, an appropriate combination of cancellation operations must be performed for each subdomain (subsystem). Throughout the workflow, each subdomain goes through a series of interim states. As a result, the combinations of cancellation operations needed by each subdomain 
change depending on the execution point in the workflow. When a WfMS controls a combination of cancellation operations, its workflow becomes far more complex than a simple combination of forward procedures to provide network elements. To simplify the workflow, we developed a status management system (StMS) described in the next section.

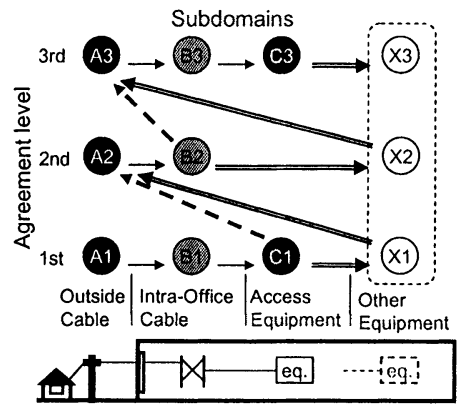

(a) ANEA workflow

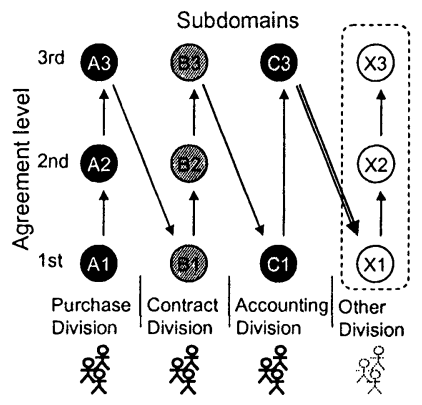

(b) Typical Business Flow
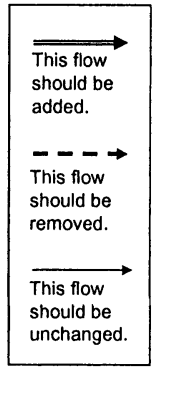

Figure 1. Comparison of the execution orders of an ANEA system and a typical business processing system and the effect of adding a new subdomain on these systems' workflow

\section{IMPLEMENTATION OF STMS}

The basic architecture of StMS is shown in Figure 2. The StMS is a middleware component that works together with the WfMS, and it exclusively handles cancellation processing. The StMS consists of two parts, a working space and a rule accumulator. The working space holds two categories of information as nodes in a graph structure shown in the left part of Figure 2. These categories show the execution history of forward operations of the subsystems and the execution schedule for cancellation operations corresponding to the forward operations. There are several links between the nodes indicating three types of relationships between the operations: dependency, (e.g., operations depend on one another) cancellation (e.g., operations have different cancellation operations), and inclusiveness (e.g., some cancellation operations can be substituted with other cancellation operations).

These links and nodes are built according to the generation rules prepared beforehand when the WfMS requests a subsystem to process an ANEA operation. The generation rules are stored in the rule accumulator. The rules have a condition part and an action part. These parts have several operation identifiers. An operation identifier indicates the type of corresponding operation, showing whether it is a forward operation or a cancellation operation. It is embodied as a unique value in the whole system. For example, an operation identifier can be coded as variable length character data or variable length integer data. The operation identifiers in the condition part of a rule indicate the nodes that the StMS should apply the rule to. The operation identifiers in the action part of a rule indicate the nodes that the StMS should look for or create. The rules holding a forward operation identifier in their condition part have several forward operation identifiers and several cancellation operation identifiers in their action part. The forward operation identifiers are used to create dependency links. The cancellation operation identifiers are used to create 
cancellation links. In contrast, the rules holding a cancellation operation identifier in their condition part have only cancellation operation identifiers in their action part. These rules are used to create inclusive links.

This basic StMS composition makes it possible to explicitly isolate cancellation processes from the forward operations in the workflow. We simulated this system and found that when both the WfMS and the StMS are used for an ANEA domain, the total number of activity nodes on 18 workflows can be reduced from 51 to 26 (Figure 3).

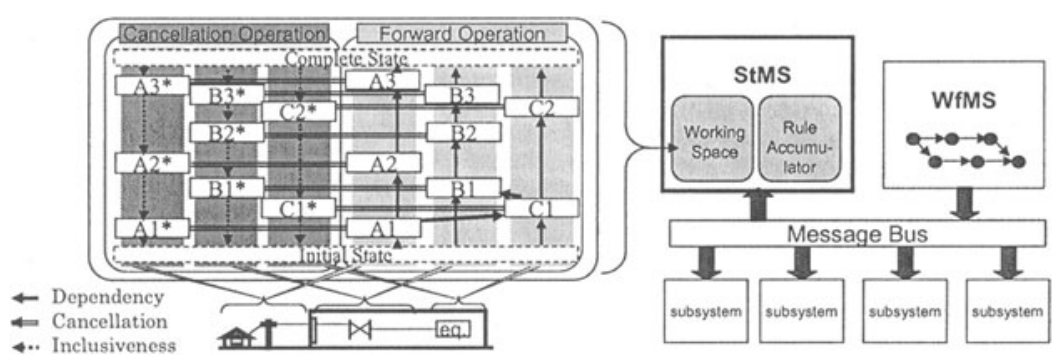

Figure 2. Basic architecture and working space in the StMS

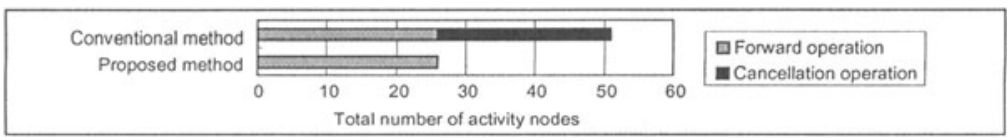

Figure 3. Total number of activity nodes on 18 workflows

\section{SUMMARY}

We analyzed the causes of complexity in the workflow of telecom operation support systems and clarified their workflow processing on the basis of a heterogeneous domain model. We then proposed a rule-driven method for automatic cancellation processing in this domain model, and described its implementation in the form of a status management system. The proposed method can reduce the total number of activity nodes by $51 \%$.

\section{REFERENCES}

[1] V.P. Wade, and T. Richardson, "Workflow - A Unifying Technology for Operational Support Systems", Network Operations and management Symposium, Vol. 07, pp. 231246, Sep. 2000.

[2] N. Edelweiss, and M. Nicolao, "Workflow modeling: exception and failure handling representation", Computer Science, 1998. SCCC '98. XVIII International Conference of the Chilean Society of, 1998, pp. 58 -67. 